\title{
VECTOR QUANTIZATION TECHNIQUES FOR MULTIPLE-ANTENNA CHANNEL INFORMATION FEEDBACK
}

\author{
June Chul Roh and Bhaskar D. Rao \\ Department of Electrical and Computer Engineering \\ University of California, San Diego \\ La Jolla, CA 92093-0407, USA \\ Email: jroh@ece.ucsd.edu, brao@ece.ucsd.edu
}

\begin{abstract}
This paper presents vector quantization (VQ) techniques in the context of multiple-antenna systems with finite-rate feedback. For MISO systems, we introduce a new design criterion and develop the corresponding iterative design algorithm for quantization of the beamforming vector. For complexity-limited systems, tree-structured VQ is also examined and compared with the full-search VQ method. The method is extended to MIMO channels by employing a parameterization method that exploits the orthonormality in the spatial information matrix and using the MISO approach in a sequential manner. The parameterization method can also be used to develop an effective low-complexity scheme to deal with quantization of time-varying channels. Also briefly discussed is a matrix quantization method for feeding back the beamforming matrix in MIMO spatial multiplexing systems.
\end{abstract}

\section{INTRODUCTION}

In recent years, multiple-antenna communication systems have been studied intensively because of the potential improvements in data transmission rates and/or link reliability. The performance achievable using multiple antennas depends on the nature of channel state information (CSI) available at the transmitter and at the receiver. We assume perfect CSI at the receiver and focus on the CSI at the transmitter (CSIT). When the transmitter has perfect CSIT, a higher capacity link can be achieved in the single user case, and there are other benefits such as lower complexity receivers and better system throughput in a multiuser environment. However, the assumption that the transmitter has perfect knowledge of the multi-dimensional channel is unrealistic as in many practical systems the channel information is provided to the transmitter through a finite-rate feedback channel. In this paper, we focus on the quantization of multipleantenna channel information with a finite number of bits. Many interesting feedback methods have been suggested and evaluated mainly through simulations. Only recently more systematic methods to quantify analytically the performance of finite-rate feedback systems have begun to appear [1], [2]. Our work is along these lines of inquiry and attempts to further the design methodology and analytical understanding of quantized beamforming systems.

This research was supported in part by UC Discovery Grant No. core02 10109 sponsored by Ericsson, and in part by the U.S. Army Research Office under MURI Grant No. W911NF-04-1-0224.
We use the following notations. $A^{\dagger}$ indicates the conjugate transpose of matrix $A$. The inner product between two vectors is defined as $\langle u, v\rangle=u^{\dagger} v$ and the 2-norm of vector $v$ is denoted by $\|v\|=\langle v, v\rangle^{1 / 2} \cdot \tilde{\mathcal{N}}(\mu, \Sigma)$ is the proper complex Gaussian random vector with mean $\mu$ and covariance $\Sigma$. Uniform distribution over a set $S$ is denoted by $\mathcal{U}(S)$. The function $\log (\cdot)$ is the natural logarithm.

\section{BEAMFORMING VECTOR QUANTIZATION FOR MISO CHANNELS}

For a MISO channel, the channel information that we want to feed back is a complex unit-norm beamforming vector. We treat the problem as a vector quantization (VQ) problem, a subject that has received much attention in source coding [3]. The often employed mean squared error (MSE) criterion in source coding, though convenient for quantization design, is not very suitable in this context because the more appropriate criteria for communication systems is channel capacity or signal-to-noise-ratio (SNR). For effective quantizer design, in this section we propose a new design criterion, namely, maximizing the mean squared weighted inner-product (MSwIP) which is directly related to channel capacity.

\section{A. System Model}

We consider a MISO system with $t$ antennas at the transmitter. Assuming flat-fading, the multiple-antenna channel is modeled by the channel vector $h \in \mathbb{C}^{t}$. That is, the channel input $x \in \mathbb{C}^{t}$ and the channel output $y \in \mathbb{C}$ have the following relationship:

$$
y=h^{\dagger} x+\eta
$$

where $\eta$ is the additive white Gaussian noise distributed by $\tilde{\mathcal{N}}(0,1)$. The average transmit power is denoted by $P_{T}$, i.e., $E\left[x^{\dagger} x\right]=P_{T}$. The channel vector will be also written in term of its magnitude and direction as $h=\alpha v$, where $\alpha=\|h\|$ and $v=h /\|h\| .^{1}$

We assume that perfect channel information is known to the receiver. It is well-known that, with perfect CSIT, transmit beamforming along $v$ is the optimum choice for maximizing the received SNR and also the mutual information. In this section, we consider quantization of the unit-norm vector for

\footnotetext{
${ }^{1}$ Though not explicitly indicated for notational simplicity, note that throughout the paper $\alpha$ and $v$ (also $\hat{v}$ later) are functions of $h$.
} 
feedback to the transmitter through a feedback channel. The capacity of the feedback channel is assumed to be finite and limited to $B$ bits per channel update. A quantization codebook $\mathcal{C}=\left\{\hat{v}_{1}, \ldots, \hat{v}_{N}\right\},\left\|\hat{v}_{i}\right\|=1, N=2^{B}$, is known to both the receiver and the transmitter. Based on the channel information, the receiver selects the best beamforming vector, say $\hat{v}$, from the codebook and sends the corresponding index for the selected beamforming vector to the transmitter. We assume feedback with no error and no delay, and focus on the design of the quantizer, i.e., codebook.

At the transmitter, $\hat{v}$ is employed as transmit beamforming vector $(\|\hat{v}\|=1)$. That is, an information-bearing symbol $s \in$ $\mathbb{C}$ is transmitted as $x=\hat{v} s$, resulting in

$$
y=\alpha\langle v, \hat{v}\rangle s+\eta
$$

where $E\left[|s|^{2}\right]=P_{T}$. In this setting, the received SNR is simply given by $\mathrm{SNR}=\alpha^{2}|\langle v, \hat{v}\rangle|^{2} P_{T}$. And the mutual information between $s$ and $y$ for given $h$ and $\hat{v}$ is given by

$$
I(h, \hat{v})=\log \left(1+\alpha^{2}|\langle v, \hat{v}\rangle|^{2} P_{T}\right)
$$

in nats per channel use, where it is assumed that $s \sim$ $\tilde{\mathcal{N}}\left(0, P_{T}\right)$. It is obvious that $I(h, \hat{v})$ with finite $B$ is less than the mutual information with perfect feedback (with $B=\infty$ )

$$
I(h, v)=\log \left(1+\alpha^{2} P_{T}\right)
$$

since $|\langle v, \hat{v}\rangle|^{2}$ is always less than one.

The capacity loss due to quantization of beamforming vector is given by

$$
\begin{aligned}
C_{L} & =E[I(h, v)]-E[I(h, \hat{v})] \\
& =E\left[I_{L}(h, \hat{v})\right]
\end{aligned}
$$

where $E[I(h, v)]$ and $E[I(h, \hat{v})]$ are the ergodic capacities when the transmitter uses the optimum and the quantized beamforming vector, respectively; and $I_{L}(h, \hat{v})=I(h, v)-$ $I(h, \hat{v})$. With a little manipulation, it can be rewritten as

$$
I_{L}(h, \hat{v})=-\log \left(1-\frac{\alpha^{2} P_{T}}{1+\alpha^{2} P_{T}}\left(1-|\langle v, \hat{v}\rangle|^{2}\right)\right) .
$$

\section{B. The MSwIP Criterion and VQ Design Algorithm}

For designing a quantizer for the beamforming vector, a good design criterion could be maximizing the expected mutual information.

$$
\max _{\mathcal{Q}(\cdot)} E \log \left(1+|\langle h, \mathcal{Q}(h)\rangle|^{2} P_{T}\right)
$$

where $\hat{v}=\mathcal{Q}(h)$ is the quantized beamforming vector $(\|\hat{v}\|=$ 1). The design criterion of (7) is equivalent to minimizing the capacity loss defined in (5). However, unfortunately the design criterion does not lead to an iterative quantizer design algorithm with monotonic convergence property ${ }^{2}$, since generally there is no analytical expression for the optimum code vector as a function of a given quantization cell [1]. In order to avoid

\footnotetext{
${ }^{2}$ Monotonic convergence means here that an improved design is guaranteed at every iteration.
}

the convergence problem, instead of using the direct form of the capacity loss, we first consider an approximation to the capacity loss. We return to the design based on (7) later and develop an iterative design method in Section II-D.

When $P_{T} \ll 1$, or when $|\langle v, \hat{v}\rangle|$ is close to one (which is valid when $N$ is reasonably large),

$$
C_{L} \simeq E\left[\tilde{\alpha}^{2}\left(1-|\langle v, \hat{v}\rangle|^{2}\right)\right]
$$

where $\tilde{\alpha}=\sqrt{\frac{\alpha^{2} P_{T}}{1+\alpha^{2} P_{T}}}$. This is a result of the fact that under the above conditions, (6) can be approximated as $I_{L}(h, \hat{v}) \simeq \tilde{\alpha}^{2}\left(1-|\langle v, \hat{v}\rangle|^{2}\right)$ using $-\log (1-x) \simeq x$ for small $x$. This approximation leads to the following quantizer design criterion.

New Design Criterion: Design a quantizer to maximize the mean squared weighted inner-product (MSwIP),

$$
\max _{\mathcal{Q}(\cdot)} E|\langle\tilde{\alpha} v, \mathcal{Q}(h)\rangle|^{2}
$$

where $\hat{v}=\mathcal{Q}(h)$ is the quantized beamforming vector $(\|\hat{v}\|=1)$. This VQ design criterion will be called the MSwIP criterion.

One of virtues with the MSwIP criterion is that it does lead to a closed-form VQ design algorithm. The design algorithm is similar in nature to the Lloyd algorithm in VQ study which is based on two conditions: the nearest neighborhood condition (NNC) and the centroid condition (CC) [3]. The same approach is used here for designing the quantizer.

New Design Algorithm:

1. NNC: For given code vectors $\left\{\hat{v}_{i} ; i=1, \ldots, N\right\}$, the optimum partition cells satisfy that for $i=1, \ldots, N$,

$$
\mathcal{R}_{i}=\left\{h \in \mathbb{C}^{t}:\left|\left\langle\tilde{\alpha} v, \hat{v}_{i}\right\rangle\right| \geq\left|\left\langle\tilde{\alpha} v, \hat{v}_{j}\right\rangle\right|, \forall j \neq i\right\} .
$$

where $\mathcal{R}_{i}$ is the partition cell (Voronio region) for the $i$ th code vector $\hat{v}_{i} . \tilde{\alpha}$ does not impact the partitioning.

2. $C C$ : For a given partition $\left\{\mathcal{R}_{i} ; i=1, \ldots, N\right\}$, the optimum code vectors satisfy that for $i=1, \ldots, N$,

$$
\hat{v}_{i}=\arg \max _{\hat{v} \in \mathcal{R}_{i},\|\hat{v}\|=1} E\left[|\langle\tilde{\alpha} v, \hat{v}\rangle|^{2} \mid h \in \mathcal{R}_{i}\right] .
$$

Since $E\left[|\langle\tilde{\alpha} v, \hat{v}\rangle|^{2} \mid h \in \mathcal{R}_{i}\right]=\hat{v}^{\dagger} E\left[\tilde{\alpha}^{2} v v^{\dagger} \mid h \in \mathcal{R}_{i}\right] \hat{v}$, the solution for the above optimization problem is

$$
\hat{v}_{i}=\text { (principal eigenvector) of } E\left[\tilde{\alpha}^{2} v v^{\dagger} \mid v \in \mathcal{R}_{i}\right] \text {. }
$$

The above two conditions are iterated until $E|\langle\tilde{\alpha} v, \mathcal{Q}(h)\rangle|^{2}$ converges. In practice, a quantizer is designed using a sufficiently large number of training samples (channel realizations). In that case, the statistical correlation matrix in (12) is replaced with a sample average.

Beamforming Vector Selection (Encoding): For a given codebook $\mathcal{C}=\left\{\hat{v}_{1}, \ldots, \hat{v}_{N}\right\}$, the receiver encodes as follows ${ }^{3}$

$$
\hat{v}=\mathcal{Q}(v)=\arg \max _{\hat{v}_{i} \in \mathcal{C}}\left|\left\langle v, \hat{v}_{i}\right\rangle\right|
$$

\footnotetext{
${ }^{3}$ Encoding process in codebook designing is denoted by $\mathcal{Q}(v)$ since it only depends on $v$, while in designing a codebook in (9) the encoding is also dependent on $\alpha$, hence, it is denoted by $\mathcal{Q}(h)$.
} 
By the encoding scheme, the unit-norm hypersphere $\mathcal{S}_{t}=$ $\left\{u \in \mathbb{C}^{t}:\|u\|=1\right\}$, where random vector $v$ lies, is partitioned into $\left\{\overline{\mathcal{R}}_{i} ; i=1, \ldots, N\right\}$, where

$$
\overline{\mathcal{R}}_{i}=\left\{v \in \mathcal{S}_{t}:\left|\left\langle v, \hat{v}_{i}\right\rangle\right| \geq\left|\left\langle v, \hat{v}_{j}\right\rangle\right|, \forall j \neq i\right\} .
$$

The encoding scheme (13) can be restated simply as $\mathcal{Q}(v)=$ $\hat{v}_{i}$ if $v \in \overline{\mathcal{R}}_{i}$.

\section{Two Related Design Methods}

The MSwIP design method is general and can be applied even for spatially correlated channels. It should be pointed out that, with the MSwIP criterion, generally the quantizer is optimized for a particular SNR (or $P_{T}$ ). As a result, we may need more than one codebooks especially if the system has multiple operating SNR points. ${ }^{4}$ Therefore, it would be interesting to find other design methods that do not depend on $P_{T}$.

As the SNR increases $\left(P_{T} \rightarrow \infty\right), \tilde{\alpha} \rightarrow 1$; hence, the MSwIP criterion in (9) reduces to

$$
\max _{\mathcal{Q}(\cdot)} E|\langle v, \mathcal{Q}(v)\rangle|^{2}
$$

where $\hat{v}=\mathcal{Q}(v)$ is the quantized beamforming vector $(\|\hat{v}\|=$ 1 ). This design criterion will be called the MSIP criterion. One can see that this criterion is based only on $v$ and independent of $\alpha$ and $P_{T}$. A design algorithm corresponding to this criterion is obtained by replacing $\tilde{\alpha}$ in the NNC and the CC for the MSwIP criterion with 1.

Another connection of the MSwIP criterion can be found by considering the other extreme case. When $P_{T} \ll 1$ (in low SNR region), $\tilde{\alpha} \simeq \sqrt{P_{T}} \alpha$; hence, the original criterion in (9) becomes

$$
\max _{\mathcal{Q}(\cdot)} E|\langle h, \mathcal{Q}(h)\rangle|^{2}
$$

This design criterion is now based on the unnormalized channel vector $h$ instead of $v$. The design algorithm can be obtained by replacing $\tilde{\alpha} v$ in the $\mathrm{NNC}$ and the $\mathrm{CC}$ for the MSwIP criterion with $h$. This VQ design method was proposed and studied by Narula et. al. [1]. Note that the design objective also can be said to be maximizing the expected received SNR.

It should be pointed out that when the channel has i.i.d. entries, i.e., $h \sim \tilde{\mathcal{N}}\left(0, I_{t}\right)$, the three design methods are equivalent, all maximizing $E[\mathrm{SNR}]$ or $E|\langle v, \hat{v}\rangle|^{2}$. Therefore, one can use the MSIP method. This is a consequence of the fact that for random channel $h \sim \tilde{\mathcal{N}}\left(0, I_{t}\right), v=h /\|h\|$ is independent of $\alpha=\|h\|$ and uniformly distributed over the unit-norm sphere $\mathcal{S}_{t}$. However, for general spatially-correlated channels, the three criterions are different since now $\alpha$ and $v$ are not independent.

\footnotetext{
${ }^{4}$ The method can be readily adapted to variability in SNR.
}

\section{VQ Design Method based on Maximizing Capacity}

We now consider a VQ design method that is targeted to maximizing the average mutual information, i.e., the criterion in (7).

\section{Design Algorithm:}

1. $N N C$ : For given code vectors $\left\{\hat{v}_{i} ; i=1, \ldots, N\right\}$, redefine the partition cells based on the mutual information measure. This results in the same NNC (10) as for the MSwIP criterion.

2. $C C$ : For a given partition $\left\{\mathcal{R}_{i} ; i=1, \ldots, N\right\}$, the optimum code vectors satisfy for $i=1, \ldots, N$,

$$
\hat{v}_{i}=\arg \max _{\hat{v} \in \mathcal{R}_{i}} E\left[\log \left(1+|\langle h, \hat{v}\rangle|^{2} P_{T}\right) \mid h \in \mathcal{R}_{i}\right] .
$$

In the context of VQ design from training channel samples, assume $K_{i}$ samples $\left\{h_{k}\right\}_{k=1}^{K_{i}}$ lie in the partition cell $\mathcal{R}_{i}$. To fine the $\mathrm{CC}$ solution, we define a Lagrangian:

$$
J=\sum_{k=1}^{K_{i}} \log \left(1+\hat{v}^{\dagger} R_{k} \hat{v}\right)-\lambda\left(\hat{v}^{\dagger} \hat{v}-1\right)
$$

where $R_{k}=P_{T} h_{k} h_{k}^{\dagger}$ and $-\lambda$ is the Lagrange multiplier. From $\partial J / \partial \hat{v}^{*}=0$, we have

$$
\left[\sum_{k=1}^{K_{i}} \frac{R_{k}}{1+\hat{v}^{\dagger} R_{k} \hat{v}}\right] \hat{v}=\lambda \hat{v} .
$$

The solution for (18) is not straightforward since $\hat{v}$ itself is embedded in the matrix expression inside the bracket. We consider the following iterative method for the $\mathrm{CC}$ solution.

$\hat{v}_{[n]}=$ (principal eigenvector) of $\left[\sum_{k=1}^{K_{i}} \frac{R_{k}}{1+\hat{v}_{[n-1]}^{\dagger} R_{k} \hat{v}_{[n-1]}}\right]$

where $\hat{v}_{[n]}$ is the solution after the $n$th iteration and $\hat{v}_{[0]}$ is the initial choice. The iteration (19) is terminated if the design objective converges or decreases compared with the previous iteration (note that monotonic convergence is not guaranteed).

The codebook obtained through the MSwIP method was used as the initial code points for the above VQ design algorithm that is based on mutual information objective. The results show that the improvement from the additional step is almost negligible, and suggests that the MSwIP design method provides almost optimal codebooks also in the sense of maximizing the average mutual information.

\section{E. Design Examples and Tree-Structured VQ}

With the MSwIP design algorithm, we can obtain an optimum codebook for any number of transmit antennas and any codebook size $N$. The performances of quantizer codebooks designed with the MSwIP design methods are shown in Fig. 1 in terms of the ergodic channel capacity. For ease of comparison, all the capacities were normalized with respect to that of the complete CSIT $\left(C_{\text {Full }}\right)$. The channel is spatially correlated with the correlation model in [4]: A linear antenna array is simulated with antenna spacing of half 
wavelength and uniform angular-spread of $\left[-30^{\circ}, 30^{\circ}\right]$. As expected, it turns out that in high SNR region the MSIP method performs better than Narula's method, and in low SNR region the reverse relation holds. Moreover, the MSwIP method always performs better than or equal to any of the other two methods in the all SNR range. The performance difference decreases as $B$ increases.

We are also interested in quantifying the effect of quantization of beamforming vector with a finite number of bits on the channel capacity. In [5], we analyzed the performance for the MISO channel with i.i.d. entries using a simple quantization-cell approximation for tractable analysis. The analytical framework is fairly general and we were able to obtain results for various performance measures including the capacity loss, the outage probability and the symbol error probability.

It is well-known that using VQ is the optimal way in representing a signal vector. However, the computational complexity can be high as searching the best code vector from the codebook is proportional to the number of code vectors. On the other hand, the VQ framework is quite rich and provides for a variety of tradeoffs. For complexity-limited systems, we can consider various suboptimal VQ methods that have been already studied for source coding [3]. Treestructured VQ (TSVQ) is one effective technique for reducing the search complexity (but with more storage). For a TSVQ of $q$-ary tree with $d$ stages (codebook size $N=q^{d}$ ), the total search complexity is proportional to $q d$ rather than $q^{d}$ in the ordinary full-search VQ. Fig. 2 shows the performance of a binary TSVQ $(q=2)$ for beamforming-vector feedback of MISO channels $(t=2,3,4)$. Same design procedure as in [3, Sec. 12.4] was employed except that in each stage our MSwIP VQ design method was used. From simulation results, we can see that there is some performance degradation compared with the full-search VQ. For example, when $t=4$ and $B=7$ the performance loss is about 1 bit.

\section{BEAMFORMING MATRIX QUANTIZATION FOR MIMO CHANNELS}

A general MIMO channel has multiple orthonormal vectors as channel spatial information for quantization. We briefly discuss ways of extending the MISO methods to the MIMO context.

\section{A. System Model}

We consider a MIMO system with $t$ transmit and $r$ receive antennas. Now the channel is modeled by the channel matrix $H \in \mathbb{C}^{r \times t}$. That is, the channel input $x \in \mathbb{C}^{t}$ and the channel output $y \in \mathbb{C}^{r}$ have the following relationship:

$$
y=H x+\eta
$$

where $\eta$ is the additive white Gaussian noise vector distributed by $\tilde{\mathcal{N}}\left(0, I_{r}\right)$. We denote the rank of $H$ by $m$. And, the singular value decomposition (SVD) of $H$ is given by $H=U_{H} \Sigma_{H} V_{H}^{\dagger}$, where $U_{H} \in \mathbb{C}^{r \times r}$ and $V_{H} \in \mathbb{C}^{t \times t}$ are unitary matrices and
$\Sigma_{H} \in \mathbb{R}^{r \times t}$ contains the singular values $\sigma_{1} \geq \ldots \geq \sigma_{m}>0$ of $H$. The transmit signal has the average transmit power $E\left[x^{\dagger} x\right]=P_{T}$.

The transmit beamforming along the first principal eigenvectors of $H^{\dagger} H$ is the optimum choice in capacity sense. Therefore, the first $n(1 \leq n \leq m)$ column vectors of $V_{H}$ are quantized for feeding back to the transmitter. For notational convenience, let us denote the $i$ th column vector of $V_{H}$ by $v_{i}$ and define the first $n$ column vectors of $V_{H}$ as $V$, i.e., $V=\left[v_{1}, \ldots, v_{n}\right]$.

The quantized matrix $\hat{V}=\mathcal{Q}(V)$ is employed as a transmit beamforming matrix. That is, an information-bearing symbol vector $s=\left[s_{1}, \ldots, s_{n}\right]^{T}$ is transmitted through $x=\hat{V} s$, resulting in

$$
y=H \hat{V} s+\eta
$$

Here we assume that $s \sim \tilde{\mathcal{N}}(0, \Phi)$ and $\Phi=P_{T}$. $\operatorname{diag}\left(\gamma_{1}, \ldots, \gamma_{n}\right)$ with $\gamma_{i} \geq 0$ and $\sum_{i} \gamma_{i}=1$. The vector $\gamma=\left[\gamma_{1}, \ldots, \gamma_{n}\right]$ will be called power allocation information.

\section{B. Sequential Vector Quantization}

Here, we summarize the key results that lead to the proposed quantization method along with the method itself. For more details, refer to [6].

1) An orthonormal-column matrix $V \in \mathbb{C}^{t \times n}$ can be uniquely represented by a set of unit-norm vectors with different dimensions, $q_{1} \in \mathcal{S}_{t}, q_{2} \in \mathcal{S}_{t-1}, \ldots, q_{n} \in \mathcal{S}_{t-n+1}$, where $\mathcal{S}_{t}=\left\{u \in \mathbb{C}^{t}:\|u\|=1\right\}$.

2) For random channel $H$ with i.i.d. $\tilde{\mathcal{N}}(0,1)$ entries, each unit-norm vector is uniformly distributed over the corresponding unit-norm sphere, i.e., $q_{i} \sim \mathcal{U}\left(\mathcal{S}_{t-i+1}\right)$ for $i=1, \ldots, n$. Furthermore, the unit-norm vectors $q_{i}$ are statistically independent.

3) For $i=1, \ldots, n$, the unit-norm vector $q_{i}$ is quantized using a codebook $\mathcal{C}_{i}$ that is designed for random unit-norm vector in $\mathbb{C}^{t-i+1}$ with the MSIP criterion.

The basic idea of the sequential quantization method is to quantize unit-norm vectors $\left\{q_{i}\right\}$ independently using a set of codebooks $\left\{\mathcal{C}_{i}\right\}$. The codebook $\mathcal{C}_{i}$ is designed for random unit-norm vector in $\mathcal{S}_{t-i+1}$ with the MSIP criterion. The independence of the unit-norm vectors $\left\{q_{i}\right\}$ indicates that the overall loss is minimal compared to joint quantization of $q_{i}$.

The multiple spatial channels in MIMO channels have different contributions to the channel capacity because each spatial channel has a different channel gain. This also has to be taken into account while designing a quantizer, leading to a problem of bit allocation.

\section{Matrix Quantization Method}

In feeding back the beamforming matrix, a more efficient approach is to jointly quantize the multiple beamforming vectors. We can extend the idea and the methodology of the MSwIP VQ design method (for MISO systems) to the problem of transmit beamforming for MIMO spatial multiplexing (SM) systems. Due to space limitations, we only 
summarize the results and details can be found in [7]. Assuming a fixed number of spatial channels and equal power allocation, we propose a new design criterion for designing the codebook of beamforming matrices that is based on minimizing the capacity loss resulting from the limited rate in the feedback channel. This can be viewed as an extension of the MISO formulation. Using the criterion, an iterative design algorithm that converges to an optimum codebook is developed. Under the i.i.d. channel and high SNR assumption, the effect on channel capacity of the finite-bit representation of beamforming matrix is analyzed. Central to this analysis is the complex multivariate beta distribution and tractable approximations to the Voronoi regions associated with the codepoints. Furthermore, to compensate for the degradation due to the equal power allocation assumption, a multi-mode SM transmission strategy is used wherein the number of data streams is determined based on the average SNR. This approach is shown to allow for effective utilization of the feedback bits.

\section{Feedback Method for Slowly Time-Varying Channels}

For slowly time-varying MIMO channels, we proposed a simple and practical quantization scheme based on parameterization [8]. A minimal number of scalar parameters are extracted from the spatial information matrix by exploiting the orthonormality in the spatial information matrix. In slowly time-varying channels, the parameters are also found to be smoothly changing over time. This observation motivated a simple feedback scheme: Each parameter is independently quantized using a simple adaptive delta modulation. The results show that the proposed feedback scheme has a channel tracking feature and achieves a capacity very close to the perfect feedback case at a reasonable feedback rate.

\section{SUMMARY}

We introduced several new vector quantization techniques for feeding backing multiple-antenna channel information through a finite-rate feedback channel. For MISO systems, we proposed a new design criterion, namely, maximizing mean squared weighted inner-product (MSwIP), and developed a Lloyd-type quantizer design algorithm. With the MSwIP quantizer design method, we can design the optimum beamforming codebook for arbitrary number of transmit antennas and quantization bits, and for any spatial correlation structure in channel. Then ways to extend the methodology for quantization in the context of MIMO channels were discussed. For this purpose, transformation of an orthonormal-column matrix to a set of unit-norm vectors with different dimensions is useful. The unit-norm vectors are then quantized independently using codebooks designed using the MSwIP criterion. We also briefly discussed a joint quantization method for multiple beamforming vectors in MIMO spatial multiplexing systems, and a simple and practical feedback method for slowly timevarying multiple-antenna channels.

\section{REFERENCES}

[1] A. Narula, M. J. Lopez, M. D. Trott, and G. W. Wornell, "Efficient use of side information in multiple-antenna data transmission over fading channels," IEEE J. Select. Areas Commun., vol. 16, pp. 1423-1436, Oct. 1998.

[2] K. K. Mukkavilli, A. Sabharwal, E. Erkip, and B. Aazhang, "On beamforming with finite rate feedback in multiple antenna systems," IEEE Trans. Inform. Theory, vol. 49, no. 10, pp. 2562-2579, Oct. 2003.

[3] A. Gersho and R. M. Gray, Vector Quantization and Signal Compression. Kluwer Academic, 1992.

[4] J. Salz and J. H. Winters, "Effect of fading correlation on adaptive arrays in digital mobile radio," IEEE Trans. Veh. Technol., vol. 43, no. 4, pp. 1049-1057, Nov. 1994.

[5] J. C. Roh and B. D. Rao, "Transmit beamforming in multiple antenna systems with finite rate feedback: A VQ-based approach," IEEE Trans. Inform. Theory, submitted for publication.

[6] _-, "Channel feedback quantization methods for MISO and MIMO systems," in Proc. IEEE PIMRC 2004, Barcelona, Spain, Sept. 2004.

[7] — "Design and analysis of MIMO spatial multiplexing systems with quantized feedback," in preparation (journal).

[8] —, "An efficient feedback method for MIMO systems with slowly time-varying channels," in Proc. IEEE WCNC 2004, Atlanta, Georgia, USA, Mar. 2004.

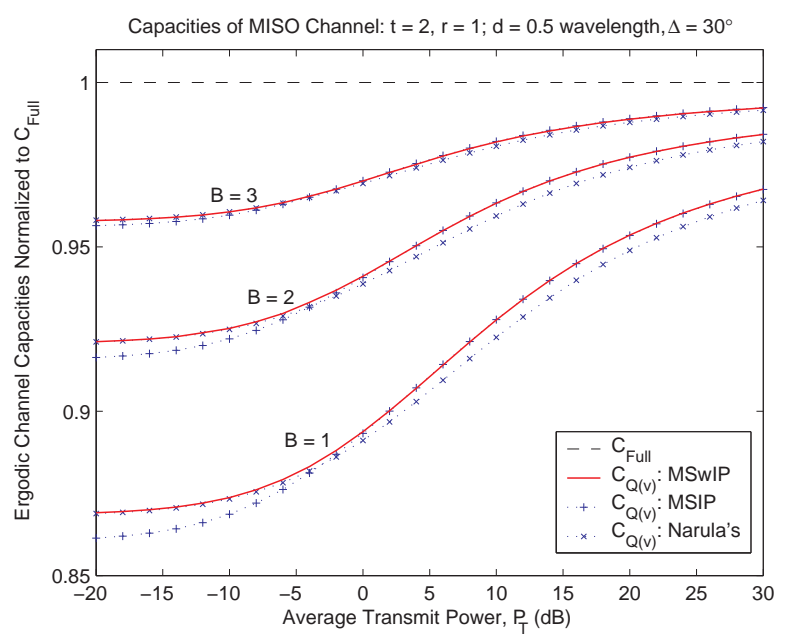

Fig. 1. Ergodic capacities of correlated MISO channels with quantized beamforming from different quantizer design methods $(t=2$ and $B=$ $1,2,3)$.

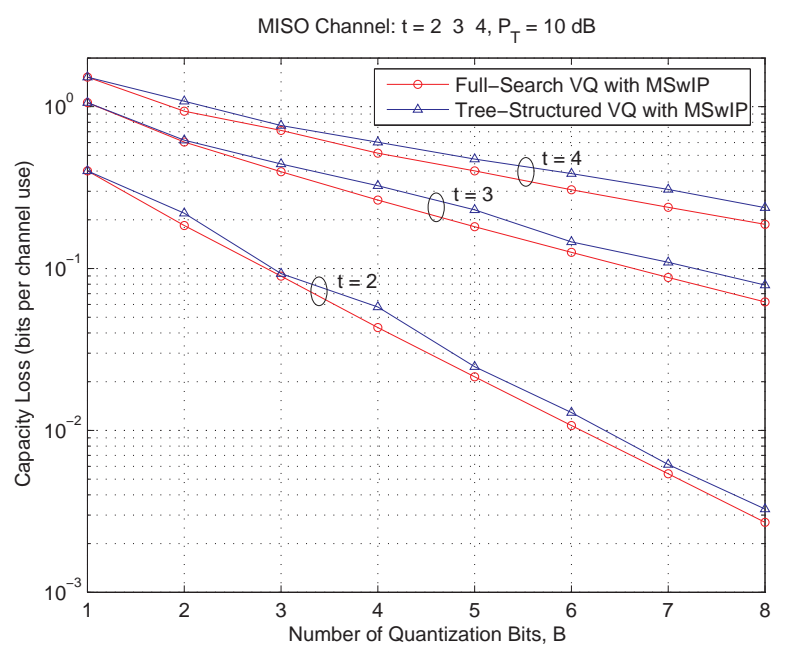

Fig. 2. Capacity loss due to quantization of beamforming vector $(t=2,3,4$; $\left.P_{T}=10 \mathrm{~dB} ; B=1,2, \ldots, 8\right)$. 